Stefano Rusconi ORCID iD: 0000-0002-0375-9990

\title{
Impact of genotypic susceptibility score on cART outcomes during primary HIV infection $\dagger$
}

A. Giacomelli1, MD, M. Fabbiani2, MD PhD, I. De Benedetto3, MD, S. Nozza4, MD, E. Focà5, MD PhD, B.M. Celesia6, MD PhD, G. Marchetti7, MD PhD, C. Mussini8, MD, A. Antinori9, MD, G. d'Ettorre10, MD PhD, G. Madeddu11, MD, A. Bandera12, MD $\mathrm{PhD}$, A. Muscatello12, MD, and S. Rusconi*1, MD, on behalf of the INACTION study group§.

1 Divisione Malattie Infettive, DIBIC Luigi Sacco, Università degli Studi di Milano; 2 Fondazione I.R.C.C.S. Policlinico San Matteo Pavia; 3 Divisione Malattie Infettive, Ospedale Amedeo di Savoia, Università di Torino; 4 Divisione Malattie Infettive, Ospedale San Raffaele IRCCS, Università Vita-Salute, Milano; 5 Divisione Malattie Infettive, Spedali Civili, Università degli Studi di Brescia; 6 U.O.C. Malattie Infettive, ARNAS Garibaldi, Catania; 7 Divisione Malattie Infettive, Dipartimento di Scienze della Salute, Università degli Studi di Milano; 8 Divisione Malattie Infettive, Università degli Studi di Modena e Reggio Emilia, Modena; 9 U.O.C. Immunodeficienze virali, Istituto Nazionale per le Malattie Infettive Lazzaro Spallanzani, IRCCS, Roma; 10 Divisione Malattie Infettive, Università degli Studi La Sapienza, Roma; 11 Divisione Malattie Infettive, Università degli Studi di Sassari, Italy; 12 Divisione Malattie Infettive IRCCS Ca’ Granda Ospedale Maggiore Policlinico, Università degli Studi di Milano.

Running head: GSS in primary HIV infection.

This article has been accepted for publication and undergone full peer review but has not been through the copyediting, typesetting, pagination and proofreading process, which may lead to differences between this version and the Version of Record. Please cite this article as doi: 10.1002/jmv.25517.

This article is protected by copyright. All rights reserved. 
* Corresponding authors

Stefano Rusconi, M.D.

Infectious Diseases Unit, DIBIC “Luigi Sacco”, University of Milan

Via G.B. Grassi, 74

20157 Milan, Italy

Phone +39.02.5031.9761

Fax +39.02 .5031 .9758$

e-mail: stefano.rusconi@unimi.it

$\S$ A complete list of clinical centers belonging to the INACTION study group is showed at the end of the manuscript.

$\dagger$ This manuscript is dedicated to Andrea De Luca M.D., a great friend, physician, mentor and scientist.

\begin{abstract}
To assess the impact of genotypic susceptibility score (GSS) on combined antiretroviral therapy (cART) outcomes during primary HIV infection (PHI) we retrospectively enrolled patients with PHI diagnosed between 2008 and 2015 at 9/24 Italian Network ACuTe HIV InfectiON (INACTION) centers. One hundred-seventy-six patients were enrolled. Fifty-five (32.9\%) patients started with more than 3 drugs and 11 (7.2\%) started with a GSS <3. Regimen’s GSS (per 1 point increase) [aOR 4.82 (95\%CI 1.62-14.28); $p=0.005]$ and baseline HIV-RNA (per $1 \log 10$ increase) [aOR 2.02 (95\%CI 1.09-3.73); $p=0.025$ ] resulted associated with early cART initiation. In conclusion, regimen's GSS resulted to be associated to the time to cART initiation during PHI.
\end{abstract}

This article is protected by copyright. All rights reserved. 


\section{Highlights}

- INACTION is an Italian network with the aim of studying PHI.

- Regimen's GSS resulted to be associated to the time to cART initiation during PHI

- No role of GSS was observed in the probability of virological control and first regimen discontinuation

- A lower GSS score was related to a X4 tropism

Key Words: GSS, HIV, primary HIV.

\section{Introduction}

Primary HIV infection (PHI) is a crucial phase of HIV infection with a limited window of opportunity ${ }^{1}$. A prompt antiretroviral treatment (cART) initiation it's able to lead to a reduction of HIV reservoir, to a better immunological recovery and to a reduction of onward HIV transmission ${ }^{2,3}$. Consequently, it is mandatory to start cART as soon as possible according to the current guidelines during $\mathrm{PHI}^{4}$. Nevertheless, the assessment of drug resistance before treatment initiation is crucial because suboptimal cART could lead to increased risk of virological failure ${ }^{5}$. The presence of transmitted drug resistance (TDR) could be a concern not only in patients with chronic HIV infection but more so in patients with PHI. The possibility that patients with PHI could be burdened by a higher rate of TDR when compared to chronically infected patients has been postulated ${ }^{6}$. Moreover, in the study by Wittkop et al. it has been observed how the presence of TDR which affects the starting regimen was associated with an increased risk of virological failure, when compared to TDR which did not affect the efficacy of the regimen ${ }^{5}$.

This article is protected by copyright. All rights reserved. 
The choice of the cART in the absence of the result of the resistance test is based on regimens with high genetic barrier. In fact, there is a possibility of transmission of viral resistant strains during $\mathrm{PHI}^{7}$ although it appears to be declining in recent years after the introduction of the integrase inhibitors ${ }^{8}$. During PHI, even though R5 viruses predominate, also $\mathrm{X} 4$ tropic viruses can be transmitted but their real impact on disease progression remains unclear 9 .

Genotypic susceptibility score (GSS) of the antiretroviral regimen built in accordance to Stanford HIV database genotypic resistance interpretation system could be a helpful tool to assess the activity of the single antiretroviral drugs composing an antiretroviral regimen ${ }^{10}$. Moreover, GSS has been demonstrated to predict the virological outcome of antiretroviral regimens $^{11,12}$.

Aim of this study was to assess the impact of baseline GSS on early cART initiation, time to virological suppression and time to first regimen discontinuation in HIV-positive patients diagnosed during PHI.

\section{Materials and Methods}

Patients with PHI diagnosed between 2008 and 2015 at 9/24 Italian Network ACuTe HIV InfectiON (INACTION) centers were enrolled in a retrospective cohort study. All patients with at least one of RT/PR/INI resistance test performed on plasma samples by Sanger technique were analyzed using the Stanford algorithm (version 8.4) and included in the final model. Estimated scores of the total antiretroviral activity of a cART regimen was analyzed with GSS. GSS drug categorization was as follow: 1 point to each drug categorized as susceptible or potential low-level resistance or low-level resistance; 0.5 point to intermediate resistance category; 0 point to the high-level resistance category. The total GSS of the regimen represents the sum of all regimen's GSS components ${ }^{10}$. In a 
subset of 60 patients GP120 V3 sequence was analyzed using the Geno2pheno algorithm. Mixed X4/DM viruses were pooled with X4 tropic viruses. Patients were included in the study if they presented at least one criterion for HIV infection diagnosis (A) and concomitantly at least one criterion for acute HIV infection (B): (A) 1) p24 reactivity, 2) HIV-RNA and/or HIV-DNA PCR detectable viral load; (B) 1) negative HIV-antibodies or low title reactivity at ELISA 3rd (or further) generation assay, 2) less than three positive bands at Western Blot test including p24, go160/gp120 or gp41, 3) less than two positive bands at RIBA test including reactivity for gp41. Patients with Fiebig stages I-V were included $^{13}$. Patients starting cART within 3 months since PHI diagnosis were classified as early cART group. Virological suppression was defined as first HIV-RNA $<50 \mathrm{cp} / \mathrm{mL}$ from the introduction of cART.

The primary end-point was to assess the impact of GSS on early cART initiation. The secondary end-points were to assess the impact of GSS on time to virological suppression and time to first regimen discontinuation.

Logistic regression analysis was performed to identify factors associated to early cART initiation. Cox regression analysis was performed to determine predictors of time to virological suppression after cART initiation and time to first-line regimen discontinuation.

$P$-value $<0.05$ was considered statistically significant. All statistical analyses were performed using SPSS version 13.0 software package (SPSS Inc., Chicago, IL, USA).

The study was approved by the Ethics Committee (EC) of Monza-Brianza for San Gerardo Hospital (coordinating center) on June 2014 and then by the EC of each participating center. Written informed consent was obtained from all enrolled subjects.

This article is protected by copyright. All rights reserved. 


\section{Results}

One hundred and seventy-six patients were enrolled. Baseline characteristics are shown in Table 1. During the study period 167 (94.9\%) of the patients started a cART regimen, of whom 125 (74.9\%) patients started during the first 3 months since HIV diagnosis. All patients who started an antiretroviral regimen during the time of observation had at least 3 drugs in their cART regimen. Fifty-five (32.9\%) patients started with more than 3 drugs. The GSS was available for 153 patients, of whom 11 (7.2\%) started with a GSS $<3$. Patients with a X4-tropic virus have lower GSS score than patients infected by a R5tropic virus (3 vs 3.5; $p=0.012$ ).

Regimen's GSS (per 1 point increase) [aOR 4.82 (95\%CI 1.62-14.28); $p=0.005]$, baseline HIV-RNA (per 1 log10 increase) [aOR 2.02 (95\%CI 1.09-3.73); $p=0.025$ ] and the calendar year 2012-2015 vs 2008-2011 [aOR 9.38 (95\%CI 3.40-25.89); $p<0.001$ ] resulted associated with early cART initiation (Table 2). No association was observed with regimen GSS and time to virological suppression during PHI (Supplementary Table 1). Regimen GSS (per 1 point increase) resulted associated to first regimen discontinuation in the univariate analysis [HR 2.21 (95\%CI 1.44-3.37); $p<0.001$ ], but the significance was lost in the multivariate model [aHR 1.15 (95\%CI 0.59-2.21); $\mathrm{p}=0.683$ ] (Supplementary Table 2).

\section{Discussion}

In our study we observed a correlation between GSS and the time of cART initiation in patients with PHI. This finding can partially be explained by the high percentage (32.9\%) of patients who started the cART with more than 3 drugs. Even though there are no current evidences favouring the start of a cART with more than three drugs ${ }^{4,14}$, many clinicians still use to start the treatment with a multitarget drug regimen waiting for the 
result of the resistance test and after that simplifying the regimen. It's possible that a multitarget drug regimen could have been chosen by the physician in charge due to the fear of TDR reported in patients acutely infected when compared to chronically infected ones $^{6,7}$. Even though, no association between first regimen GSS during PHI and time to virological control was observed in our study. A promptly revision of the cART is mandatory after the result of the resistance test to avoid the exposure to suboptimal antiretroviral regimen during PHI.

X4 tropic viruses had a GSS lower when compared to R5 tropic viruses. In a previous study an increased risk of harbouring drug resistant viruses wasn't observed for X4 tropic viruses when compared to R5 tropic viruses ${ }^{15}$. Whereas, we did not find any association between viral tropism and cART investigated outcomes, thus the impact on HIV disease progression remains to be clarified ${ }^{16}$.

Our study accounts for several limitations. First, the retrospective nature of the study could have affected the analysis due to missing data. Second, the integrase sequences were available only for 50 patients. Nevertheless, we did not observe any integrase region resistance in the 50 patients tested in our cohort; accordingly, for the GSS calculation integrase inhibitors were considered as fully susceptible as supported by literature until $2015^{17}$. This assumption could be not applicable to more recent years. In fact, due to the widespread use of integrase inhibitors, transmitted resistance during PHI could be observed $^{18}$. Third, only 60 patients had an available V3 loop sequence. Consequently, all 23 patients treated with maraviroc had an available V3 loop sequence, thus we were able to calculate their GSS.

According to our findings, regimen's GSS resulted to be associated to the time to cART initiation during PHI. Together with an expected better outcome with an earlier cART, 
the GSS evaluation by clinicians has been used prior to cART start, at least during 20082015.

\section{Bibliography}

1. Cohen MS, Shaw GM, McMichael AJ, Haynes BF. Acute HIV-1 Infection. $N$ Engl J Med. 2011;364(20):1943-54.

2. Schuetz A, Deleage C, Sereti I, et al. Initiation of ART during early acute HIV infection preserves mucosal Th17 function and reverses HIV-related immune activation. PLoS Pathog. 2014; 10:e1004543.

3. Robb ML, Ananworanich J. Lessons from acute HIV infection. Curr Opin HIV AIDS. 2016 Nov;11(6):555-560.

4. https://aidsinfo.nih.gov/guidelines/html/1/adult-and-adolescent-arv/20/acute-andrecent--early--hiv-infection

5. Wittkop L, Günthard HF, de Wolf F, et al; EuroCoord-CHAIN study group. Effect of transmitted drug resistance on virological and immunological response to initial combination antiretroviral therapy for HIV (EuroCoord-CHAIN joint project): a European multicohort study. Lancet Infect Dis. 2011;11(5):363-71.

6. Yanik EL, Napravnik S, Hurt CB, et al. Prevalence of transmitted antiretroviral drug resistance differs between acutely and chronically HIV-infected patients. $J$ Acquir Immune Defic Syndr. 2012;61(2):258-262.

7. Hofstra LM, Sauvageot N, Albert J, et al. Transmission of HIV drug resistance and the predicted effect on current first-line regimens in Europe. Clin Infect Dis. 2015;62(5):655-663.

This article is protected by copyright. All rights reserved. 
8. Frange P, Assoumou L, Descamps D, et al; French ANRS CO 6 PRIMO Cohort, the ANRS 147 OPTIPRIM Clinical Trial and the AC11 Resistance Study Groups; French ANRS CO 6 PRIMO Cohort the ANRS 147 OPTIPRIM Clinical Trial and the AC11 Resistance Study Groups. HIV-1 subtype B-infected MSM may have driven the spread of transmitted resistant strains in France in 2007-12: impact on susceptibility to first-line strategies. $J$ Antimicrob Chemother. 2015;70(7):2084-9.

9. Rieder P, Joos B, Scherrer AU, et al. Characterization of human immunodeficiency virus type 1 (HIV-1) diversity and tropism in 145 patients with primary HIV-1 infection. Clin Infect Dis. 2011;53(12):1271-9.

10. Tang MW, Liu TF, Shafer RW. The HIVdb system for HIV-1 genotypic resistance interpretation. Intervirology. 2012;55(2):98-101.

11. Rhee SY, Fessel WJ, Liu TF, et. Predictive value of HIV-1 genotypic resistance test interpretation algorithms. J Infect Dis. 2009 Aug 1;200(3):453-63.

12. Gonzalez-Serna A, Min JE, Woods C, et al. Performance of HIV-1 drug resistance testing at low-level viremia and its ability to predict future virologic outcomes and viral evolution in treatment-naive individuals. Clin Infect Dis. 2014 Apr;58(8):1165-73.

13. Fiebig EW, Wright DJ, Rawal BD, et al. Dynamics of HIV viremia and antibody seroconversion in plasma donors: implications for diagnosis and staging of primary HIV infection. AIDS. 2003;17(13):1871-9.

14. Chéret A, Nembot G, Mélard A et al. Intensive five-drug antiretroviral therapy regimen versus standard triple-drug therapy during primary HIV-1 infection 
(OPTIPRIM-ANRS 147): A randomised, open-label, phase 3 trial. Lancet Infect Dis. 2015;15(4):387-96.

15. Frange P, Galimand J, Goujard C, et al. High frequency of X4/DM-tropic viruses in PBMC samples from patients with primary HIV-1 subtype-B infection in 1996-2007: the French ANRS CO06 PRIMO Cohort Study. J Antimicrob Chemother. 2009;64:135-141.

16. Ghosn J, Galimand J, Raymond S, et al; ANRS CO 06 PRIMO cohort. X4 tropic multi-drug resistant quasi-species detected at the time of primary HIV-1 infection remain exclusive or at least dominant far from PHI. PLoS One. 2011;6(8):e23301.

17. Stekler JD, McKernan J, Milne R et al. Lack of resistance to integrase inhibitors among antiretroviral-naïve subjects with primary HIV-1 infection, 2007-2013. Antivir Ther. 2015; 20:77-80.

18. Ambrosioni J, Nicolás D, Manzardo C, et al. Integrase strand-transfer inhibitor polymorphic and accessory resistance substitutions in patients with acute/recent HIV infection. J Antimicrob Chemother. 2017;72(1):205-209.

\section{Experimental Ethics}

The study was approved by the Ethics Committee (EC) of Monza-Brianza for San Gerardo Hospital (coordinating center) on June 2014 and then by the EC of each participating center. Written informed consent was obtained from all enrolled subjects.

\section{Disclosure of Conflicts of interest}

A.G. and I.d.B. do not declare any competing interest. M.F. received speakers’ honoraria and support for travel to meetings from Bristol-Myers Squibb, Gilead, Merck Sharp \& 
Dohme, ViiV Healtcare, Janssen-Cilag, and fees for attending advisory boards from Bristol-Myers Squibb and Gilead. S.N. received travel grants and fees for presentations from ViiV, Gilead and Janssen. E.F. received consultancy fees and speaker's honoraria from, Gilead, ViiV Healthcare, Janssen-Cilag and Merck Sharp \& Dohme. B.M.C. received grants, travel grants and speaker's honoraria from Abbvie, Bristol-Myers Squibb, Gilead, ViiV, Janssen-Cilag and Merck Sharp \& Dohme. G.M. received personal fees from Gilead Sciences and ViiV Healthcare. C.M. participated to Advisory boards supported by Gilead, ViiV, BMS, janssen, MSD, Angelini, Abbvie, received grants from ViiV, MSD, Gilead, Janssen and participated to speaker's bureau supported by Gilead. A.A received grants, personal fees and non-financial support from Gilead Sciences, ViiV Healthcare and Bristol Myers Squibb, grants and personal fees from Janssen-Cilag, personal fees from Merck Sharp and Dohme and personal fees and non-financial support from Abbvie. G.d'E. received consultancy payments and speaking fee from BristolMyers Squibb, Gilead, ViiV Healthcare, Merck Sharp Dohme, ABBvie and Janssen. G.M. received consultancy and/or speakers' fees from Abbott, Bristol Myers Squibb, Gilead Sciences, Janssen, Merck Sharp \& Dohme and ViiV. A.B. received consultancy payments, speaking fees, travel and research grants from Bristol-Myers Squibb, Gilead, ViiV Healthcare, Merck Sharp Dohme, ABBvie and Janssen. A.M. received consultancy payments, speaking fees, travel and research grants from Bristol-Myers Squibb, Gilead, ViiV Healthcare, Merck Sharp Dohme, ABBvie and Janssen. S.R. received consultancy payments, speaking fees, travel and research grants from Bristol-Myers Squibb, Gilead, ViiV Healthcare, Merck Sharp Dohme, ABBvie and Janssen.

This article is protected by copyright. All rights reserved. 


\section{Acknowledgements}

We thank all the clinicians and patients involved in the INACTION study.

\section{INACTION study group participating physicians and centers}

Andrea Gori, Antonio Muscatello, Alessandra Bandera (Ospedale Maggiore Policlinico Milano); Giuseppe Tambussi, Silvia Nozza, Marco Ripa, Raffaele Dell'Acqua (H San Raffaele Milano); Andrea Antinori, Carmela Pinnetti (INMI Spallanzani Roma); Andrea Calcagno, Giancarlo Orofino, Ilaria De Benedetto, Micol Ferrara (Torino); Cristina Mussini, Vanni Borghi, Federica Carli (Modena); Benedetto Maurizio Celesia (Catania); Lucio Cosco, Carlo Torti (Catanzaro); Gabriella d'Ettorre (Umberto I Roma); Antonio Di Biagio (Genova); Emanuele Focà, Eugenia Quiros-Roldan (Brescia); Antonina Franco (Siracusa); Diego Ripamonti, Franco Maggiolo (Bergamo); Roberto Gulminetti, Massimiliano Fabbiani (Pavia); Sandro Piga, Marzia Garau, Marco Campus (Cagliari); Stefano Rusconi, Tiziana Formenti, Arianna Gabrieli, Alessia Lai, Andrea Giacomelli, Cecilia Bonazzetti (H Sacco Milano); Giulia Marchetti, Camilla Tincati (H San Paolo Milano); Antonella Cingolani (H Gemelli Roma); Giordano Madeddu (Sassari).

\section{Tables}

Population characteristics $(\mathrm{n}=176)$ $\mathrm{N}(\%)$ or median (IQR)

Age, years $37(28-45)$

Male gender $153(86.9)$

Risk factor:

$$
\text { Heterosexual }
$$

Homo/bisexual

IDU $6(3.4)$

This article is protected by copyright. All rights reserved. 
Fiebig stage:

$\begin{array}{cc}\text { I } & 6(3.4) \\ \text { II } & 22(12.5) \\ \text { III } & 28(15.9) \\ \text { IV } & 59(33.5) \\ \text { V } & 61(34.7)\end{array}$

CDC Stage:

A

B

C

Unknown

CD4, cells/mm3

HIV-RNA, $\log 10$

First cART regimen $(n=167)$ including:

$\begin{array}{rc}\text { NRTI } & 164 / 167(98.2) \\ \text { NNRTI } & 29 / 167(17.4) \\ \text { PI } & 120 / 167(71.9) \\ \text { INSTI } & 61 / 167(36.5)\end{array}$

Entry inhibitor

Early cART initiation (within 3 months from the diagnosis)

First cART regimen $>3$ drugs

55/167 (32.9)

Available V3 loop sequence

$\mathrm{X} 4$

R5
$133(75.6)$

$15(8.5)$

$1(0.6)$

$27(15.3)$

$453(329-603)$

$5.76(5.07-6.42)$
$125 / 167(74.9)$

$60(34.1)$

$17 / 60(28.3)$

$43 / 60(71.7)$

This article is protected by copyright. All rights reserved. 
FPR\%

$27.7(9.4-61.0)$

FPR $>60$

Available integrase sequences

\%Resistance

Available RT-PR sequences

GSS first regimen $(n=153)$

GSS first regimen $<3$
$16 / 60(26.7)$

$50(28.4)$

$0 / 50(0)$

$165(93.8)$

$3(3-4)$

$11 / 153(7.2)$

Table 1. Baseline characteristics of the population.

Note: During the study period 167 (94.9\%) of the patients started a cART regimen, of whom 153 had available sequences to assess the GSS.

Abbreviations: $\mathrm{N}=$ number, $\mathrm{IQR}=$ inter quartile range, $\mathrm{IDU}=$ intravenous drug users, $\mathrm{CDC}=$ Center for Disease and Control, $\mathrm{CD}=$ cluster of differentiation, $\mathrm{cART}=$ combined antiretroviral regimen, $\mathrm{X} 4=\mathrm{CXCR} 4$ tropic virus, R5= CCR5 tropic virus, $\mathrm{RT}=$ reverse transcriptase, $\mathrm{PR}=$ protease, $\mathrm{FPR}=$ false positive rate, $\mathrm{GSS}=$ genotypic susceptibility score.

\begin{tabular}{lcccc}
\hline & $\begin{array}{c}\text { Univariate } \\
\text { analysis }\end{array}$ & & Multivariate analysis & \\
& OR (95\% CI) & p & aOR (95\% CI) & p \\
\hline $\begin{array}{l}\text { Age, years (per 10 years } \\
\text { increase) }\end{array}$ & $0.97(0.70-1.35)$ & 0.872 & & \\
\hline Gender, male vs female & $1.73(0.67-4.42)$ & 0.256 & & \\
\hline Risk factor: & & & & \\
Heterosexual & Ref & 0.021 & Ref & 0.275 \\
Homo/bisexual & $2.39(1.14-4.99)$ & 0.646 & $1.81(0.63-5.22)$ & 0.499 \\
IDU & $1.73(0.17-$ & 0.911 & $2.43(1.19-31.98)$ & 0.352 \\
Other/unknown & $17.80)$ & & $0.19(0.01-6.38)$ & \\
& $1.15(0.10-$ & & & \\
\hline
\end{tabular}

This article is protected by copyright. All rights reserved. 


\begin{tabular}{|c|c|c|c|c|}
\hline $\begin{array}{l}\text { Months from last negative HIV } \\
\text { test, (per } 1 \text { month increase) }\end{array}$ & $1.01(0.99-1.02)$ & 0.619 & & \\
\hline $\begin{array}{l}\text { Calendar year at diagnosis: } \\
\text { 2012-2015 vs 2008-2011 }\end{array}$ & $\begin{array}{l}6.53(3.05- \\
14.00)\end{array}$ & $\begin{array}{c}<0.00 \\
1\end{array}$ & $9.38(3.40-25.89)$ & $\begin{array}{c}<0.00 \\
1\end{array}$ \\
\hline Fiebig stage, IV-V vs I-III & $1.10(0.52-2.32)$ & 0.797 & & \\
\hline $\begin{array}{l}\text { Symptomatic acute HIV } \\
\text { infection }\end{array}$ & $1.99(0.72-5.52)$ & 0.186 & & \\
\hline STD & $0.83(0.25-2.79)$ & 0.758 & & \\
\hline \multicolumn{5}{|l|}{ Baseline laboratory results: } \\
\hline $\begin{array}{l}\text { CD4, cells/mm3 (per } \\
\text { 100cell/mm3 increase) }\end{array}$ & $0.89(0.76-1.05)$ & 0.171 & $0.97(0.78-1.21)$ & 0.805 \\
\hline $\begin{array}{l}\text { CD8, cells/mm3 (per } \\
\text { 1cell/mm3 increase) }\end{array}$ & $0.99(0.97-1.02)$ & 0.552 & & \\
\hline $\mathrm{CD} 4 / \mathrm{CD} 8>1$ & $0.99(0.26-3.80)$ & 0.993 & & \\
\hline $\begin{array}{l}\text { HIV-RNA, } \\
\text { per } 1 \log 10 \text { increase }\end{array}$ & $1.98(0.31-2.97)$ & 0.001 & $2.02(1.09-3.73)$ & 0.025 \\
\hline $\mathrm{X} 4$ vs R5 & $1.01(0.18-5.81)$ & 0.988 & & \\
\hline $\mathrm{FPR}>60$ vs $\mathrm{FPR}<=60$ & $0.92(0.16-5.30)$ & 0.927 & & \\
\hline $\begin{array}{l}\text { First regimen’s GSS, per } 1 \\
\text { point increase }\end{array}$ & $\begin{array}{l}5.20(2.21- \\
12.25)\end{array}$ & $\begin{array}{c}<0.00 \\
1\end{array}$ & $4.82(1.62-14.28)$ & 0.005 \\
\hline
\end{tabular}

First regimen's GSS, $<3$ vs $\geq 3 \quad 0.62(0.17-2.23) \quad 0.461$

Table 2. Factors associated to early cART initiation (logistic regression analysis).

Note: The multivariate model was built by entering in the model all variable with a $p<0.05$ in the univariate analysis and adjusting for clinically significant variables (baseline CD4 cell count). The final model was built on the 153 patients with an available GSS.

Abbreviations: $\mathrm{OR}=$ odds ratio, $\mathrm{aOR}=$ adjusted odds ratio, $\mathrm{CI}=$ confidence interval, vs= versus, IDU= intravenous drug users, $\mathrm{CD}=$ cluster of differentiation, $\mathrm{STD}=$ sexually transmitted diseases, cART= combined antiretroviral regimen, X4= CXCR4 tropic, R5= CCR5 tropic, FPR= false positive rate, GSS= genotypic susceptibility score.

This article is protected by copyright. All rights reserved. 